\title{
Formación del profesorado a través de las prácticas profesionalizadoras
}

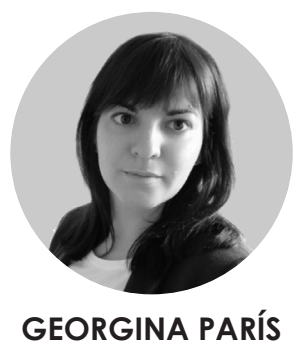

UNIVERSITAT AUTÒNOMA DE BARCELONA

georgina.paris@uab.cat

$\mathrm{L}$ a presente revisión teórica pretende explorar la dualidad de perfiles del docente cuando este interviene en un contexto de formación inicial y reglada como profesor, o por el contrario cuando actúa en un contexto laboral como formador. Aunque ambos perfiles comparten rasgos, capacidades y competencias similares, cabe destacar que los procesos formativos para su profesionalización presentan notables diferencias. Mientras los profesores cuentan con una formación prescriptiva que responde a modelos competenciales certificados por las respectivas agencias de calidad, y además existe un número importante de iniciativas que promueven experiencias innovadoras para mejorar las prácticas profesionalizadoras de su desempeño; los formadores del sistema laboral se enfrentan a condiciones más austeras. La diversidad que se mantiene en toda Europa en cuanto a la formación inicial de los profesionales del empleo dificulta la obtención de un único referente. Delimitar sus perfiles y sus competencias profesionales mejoraría su identidad profesional, permitiendo a la vez avanzar en dispositivos y modalidades de formación más profesionalizadoras.

\section{PALABRAS CLAVE:}

Profesor; formador; profesionalización; formación inicial; formación para el empleo.

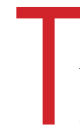
he present theoretical review attempts to explore the duality of teacher profiles when the teacher intervenes in a context of initial and regulated training as a teacher, or conversely when he acts in a work context as a trainer. Although both profiles share similar features, capacities and competences, it should be noted that the training processes for their professionalization present notable differences. While teachers have prescriptive training that responds to competency models certified by the respective quality agencies, and there are also a significant number of initiatives that promote innovative experiences to improve professional practices of their performance, trainers of the labor system face more austere conditions. The diversity that is maintained throughout Europe in terms of initial training for employment professionals makes it difficult to obtain a single reference. Defining their profiles and professional skills would improve their professional identity, while allowing progress in more professionalizing devices and training modalities.

\section{KEYWORDS:}

Teacher; trainer; professionalization; initial training; employment training. 


\section{Introducción}

a heterogeneidad de profesionales de la educación/ formación en Europa hace difícil hablar de un único perfil profesional de profesor/a. Esta diversidad puede ser explicada por múltiples factores (Tutschner y Kirpal, 2008). En primer lugar, los perfiles de profesorado son tan dispares como distintos son los Sistemas Educativos (Formación formal y reglada) y los Sistemas de Formación para el Trabajo (Formación no formal) de cada país de la Unión Europea. Cierto es que a partir de la Estrategia de Lisboa (Comisión Europea, 2000) se empezó a considerar la educación, la formación profesional para el trabajo y la cualificación como tres elementos claves al servicio del empleo. De ahí que la Unión Europea creara dos Marcos Europeos de Cualificaciones. Por un lado, el Marco de Cualificaciones del Espacio Europeo de Educación Superior (QF-EHEA) aprobado en 2005 como resultado del proceso de Bolonia. Este se refiere a los títulos del sistema de educación superior y presenta 4 niveles. Y por otro lado, el Marco Europeo de Cualificaciones para el Aprendizaje a lo Largo de la Vida (EQF) adoptado oficialmente en 2008. Este último se refiere a la formación para el empleo, y se organiza en 8 niveles.

La finalidad de este marco europeo era armonizar los distintos sistemas de la Unión Europea de cada uno de los estados miembros bajo dos lógicas: la Initial Vocational Education and Training (IVET) ${ }^{2}$ y la Continuing Vocational Education and Training $(\mathrm{CVET})^{3}$; proporcionar una forma eficaz de intercambio entre todos los profesionales así como dotar de una dimensión y de una agilidad sin precedentes al proceso de cambio emprendido entre países de un mismo continente.

Otra cuestión es la relativa a las cualificaciones y competencias profesionales exigidas a los profesores. El sistema educativo reglado regula mediante las agencias de calidad los planes formativos de los diferentes perfiles profesionales. Sin embargo, la tendencia hacia la profesionalización de los profesores de la CVET no es muy común en Europa, pues los marcos de certificación no son homogéneos y además no son prescriptivos. Pocos países exigen a sus profesionales requisitos de acceso y formación andragógica asociada a la profesión (París, 2015).

También cabe destacar la distinción entre la IVET y CVET, que no siempre es posible porque ambos sistemas se superponen generando un panorama ideal para la proliferación de múltiples perfiles profesionales. En los países anglosajones la distinción entre los dos tipos de formación no es posible ya que la formación inicial normalmente tiene lugar en situaciones que son características de la formación continua. Los países con una tradición formativa dual normalmente se organiza de forma completamente diferente. Por no hablar de los países mediterráneos, donde ambos sistemas funcionan de forma independiente (París et al., 2014).

Otro aspecto que genera controversia es pensar que el profesor emerge en un único contexto y con un único perfil. "Son múltiples las situaciones y escenarios de actuación profesional tanto dentro como fuera del sistema educativo, y a la vez múltiples los perfiles que podemos encontrar dentro de lo que podemos considerar la familia profesional de la educación, así como la formación y profesionalización de los mismos" (Tejada et al., 2007, p. 472). Este mismo autor (Tejada, 2009) dibujó los diferentes perfiles que podemos encontrar en la actualidad. Por un lado, los perfiles profesionales más comunes del sistema de formación inicial son: maestro de primaria, maestro de infantil, educador social, psicopedagogo, pedagogo, psicólogo especialista en educación, profesor de universidad y profesor de formación profesional, entre otros. Y, por otro lado, los perfiles profesionales del sistema de la formación profesional para el trabajo son: gestor de la formación, formador, orientador, formador de formadores y auditor de la formación, entre otros.

Cada uno de estos perfiles profesional representa un "conjunto de rasgos, capacidades y competencias que, certificándose por quien tiene la competencia jurídica para ello, permiten que alguien sea reconocido por la sociedad por "tal" profesional, pudiéndosele encomendar tareas para las que se le supone capacitado y competente" (Hawes y Corvalán, 2005, p. 14). Aunque cabe destacar que estos perfiles pueden desarrollar competencias y funciones profesionales distintas. Por ejemplo, el maestro de primaria puede desarrollar competencias de dirección, coordinación pedagógica, mentorazgo, docencia, evaluaciones internas y externas, etc. En el caso de los profesores de secundaria sucede lo mismo. Un profesor de secundaria puede adquirir diferentes funciones, aunque estas vendrán condicionadas por el área de experticia (área de lengua, área de matemáticas, etc.). En el supuesto de la CVET se replica el mismo ejemplo (Blignières-Légeraud, 2000; CEDEFOP, 2008, p. 176; Cort et al., 2004, p. 9; García Molina, 2013, p. 6). Mientras que un gestor de la formación aglutina competencias profesionales como la consultoría, administración, burocratización y certificación, entre otros, un formador desempeña funciones como la programación, diseño de materiales didácticos, implementación de docencia y evaluación.

\section{El profesor: un perfil profesional en la IVET}

S on muchas las voces que intentan interpretar el éxito o fracaso de la formación inicial que se les ofrece a los futuros profesores (Danielson, 2013; Fernández Enguita et al., 2010). Recientes estudios internacionales (OCDE, 2019) han puesto en evidencia los insatisfactorios resultados de aprendizaje de los alumnos en las etapas de formación obligatoria. Más allá de los resultados de los estudiantes, PISA pone interés en estudiar el perfil de los profesores y cómo su desempeño profesional facilita el aprendizaje de los alumnos. Las investigaciones confirman cómo la calidad educativa es un elemento clave para favorecer el rendimiento académico de los estudiantes. Es decir, aquel profesor que disponga de mejor formación inicial, mayor oportunidad de prácticas profesionalizadoras, de tutores cualificados para su 
mentorazgo profesionalizador, de modalidades formativas basadas en el learning by doing, entre otras, se convertirá en un profesor mejor para sus alumnos. Dicho de otra forma, los estudiantes que tengan la suerte de encontrar profesores con todo este despliegue competencial podrán aprender más y mejor (American Council on Education, 1999; Comisión Europea, 2007).

Teniendo en cuenta esta premisa, la tarea que nos precede es identificar las mejores experiencias profesionalizadoras de profesores de la IVET para ajustar la formación inicial de estos profesionales. En el Estado español, con la reforma universitaria derivada de la construcción del Espacio Europeo de Educación Superior, los estudios para ser profesor en educación infantil y primaria se han prolongado un año y a los futuros profesores de secundaria se les exige un master oficial de dos años y acreditado por las agencias de calidad sobre capacitación pedagógica. Todo ello junto a iniciativas profesionalizadoras como la formación que se ofrece en la Universidad de Lleida, concretamente en el grado de educación primaria, donde por primera vez en el Estado español se ha ofrecido la modalidad formativa dual o de alternancia (Coiduras et al., 2017), o como el proyecto PATHWAY (ARMIF: AGAUR, 2015; París et al., 2015) que evaluaba el Grado de Educación Primaria-inglés de la Universidad Autónoma de Barcelona, o como el proyecto LETEGR2 de la Universidad de Atenas (Andria, 2020; Andria y Iakovou, 2019) sobre la formación de los profesores de lenguas extranjeras, o el proyecto "PrimaryCLIL" de la Universidad de Sevilla y la Liverpool Hope University donde se establece como uno de sus objetivos "apoyarse en el Perfil Europeo del Profesorado de Lenguas Extranjeras definiendo un perfil del maestro de AICLE en educación primaria" (EUCLID: APPC, 2008), entre otros, hace indicar una mejor formación basada en el enfoque competencial y con una mayor actividad formativa de los aprendices a profesor en los centros de prácticas, escenarios imprescindibles para la adquisición de las competencias docentes en la formación inicial.

Es cierto que la formación inicial del profesorado es prescriptiva y las reformas recientes confirman la tendencia a mejorar las cualificaciones requeridas para los profesores. Sin embargo, y como apuntan Thorslund y Jacobsen (2006) y Barber y Mourshed (2007), aunque existe un acuerdo general de que es necesario aumentar la calidad de los docentes, hay menos acuerdo sobre las medidas que deben adoptarse para determinar cómo hacerlo.

\section{El formador, ¿un único perfil profesional de la CVET?}

La diversidad que se mantiene en toda Europa en cuanto a la formación inicial de los profesionales de la CVET dificulta la obtención de un único referente. Tal vez se haya subestimado la magnitud del desafío (acuerdos post-Lisboa) de poner en marcha una de las piedras angulares de la reforma a nivel europeo, diseñar la identidad profesional, las competencias profesionales, los requisitos de acceso, el desarrollo profesional y los procesos de evaluación del desempeño profesional de los profesionales. Y más teniendo en cuenta la antagónica realidad contextual de la CVET Europea, pues sólo Inglaterra, Irlanda, los países de la Benelux y Francia tienen el sistema de la CVET regulado; frente a países como Austria, República Checa, Finlandia, España, Estonia, Grecia, Islandia, Italia, Letonia, Liechtenstein, Lituania, Noruega y Suecia donde la CVET no está regulada, sin olvidar aquellos países del sudeste de Europa como Polonia, República Checa, países bálticos y Bulgaria donde la transición política y económica imposibilitó dicho proceso (Tutschner y Kirpal, 2008).

Iniciar el proceso de regularización de la CVET en Europa empieza por diseñar y exigir una formación inicial obligatoria para los profesionales de esta profesión. Cualquier oficio que carezca de la formación inicial da lugar a una profesionalización disciplinar inadecuada. Por supuesto, este es el panorama de la CVET europea. Según la información proporcionada por la Comisión Europea (Comisión Europea, "Eurypedia", 2013) sobre los 28 países que conforman la Unión Europea, incluyendo a Islandia, Suiza y Noruega, sólo Austria, Dinamarca, Francia, Hungría, Inglaterra, Italia, Malta, Luxemburgo, Rumania y Suiza exigen una formación andragógica (inicial, regulada y obligatoria) para los profesionales de la CVET. Mientras, países como Alemania, Bélgica, Bulgaria, Chipre, Eslovaquia, Eslovenia, España, Estonia, Finlandia, Grecia, Irlanda, Italia, Lituania, Noruega, Polonia, Portugal y República Checa buscan, en su mayoría, maestros de primaria, profesores de secundaria y/o formación profesional, profesionales con educación superior, o bien profesionales especialistas o expertos sobre una área de conocimiento con larga o media experiencia laboral (París et al., 2014). 


\section{Prácticas profesionalizadoras de los profesores y formadores}

E objetivo de las prácticas profesionalizadoras de los profesores de la IVET y formadores de la CVET es, en general, mejorar el conocimiento del profesorado sobre la enseñanza, los métodos pedagógicos y del uso del conocimiento en el aula real (Goldhaber y Brewerm, 2000). La formación inicial y continua y la experiencia o prácticas en el campo constituyen las dos grandes áreas de capacitación. La formación del profesorado debe comprender tanto la teoría como los aspectos prácticos de una manera integrada (Marable y Raimondi, 2007; Wilson y Ferrini-Mundy, 2001).

Varios estudios sobre programas de capacitación para profesores y formadores muestran la esencia de la integración de teoría específica en contextos de aprendizaje prácticos (Coiduras et al., 2014). La oportunidad de reflexión sobre este ejercicio profesionalizador debe acompañar el proceso, pues es en este camino donde el aprendiz a formador/profesor va construyendo su identidad profesional (Hollingsworth, 1989).

Según Sergiovanni y Starratt (2007), desarrollar estándares para profesores/formadores "efectivos" es esencial en el proceso de formación, porque los estándares actúan como un marco para definir qué es una buena práctica profesional. El marco también permite al formador/profesor localizar problemas, limitaciones y retos que pueden acontecer en su praxis. Cabe destacar, también, que las creencias y las percepciones de los propios profesores/formadores sobre sus competencias profesionales determinaran su desempeño profesional. En base a esto, Tschannen-Moran y Woolfolk Hoy $(2001$, 2007) desarrollaron y validaron la escala de eficacia de los profesores/formadores que mide tres componentes:

1) la eficacia sobre la implementación docente

2) la eficacia sobre la gestión del aula

3) la eficacia sobre la motivación del alumnado

Los resultados de sus estudios muestran que aquellos profesores/formadores con mayor experiencia profesional o que en su formación inicial han optado por modalidades formativas más prácticas y procedimentales presentan mayor nivel de eficacia en los tres componentes.

Cocca y Cocca (2016) mostraron en su estudio una comparativa entre profesores noveles y experimentados de lenguas extranjeras. Los autores hallaron diferencias en la eficacia profesional según los años de experiencia y nivel educativo, pero no en cuanto a la formación. La gran mayoría de las investigaciones coinciden en que la experiencia profesional es la fuente más influyente en el desarrollo y desempeño profesional (Morris y Usher, 2011; ReyesCruz y Gutiérrez, 2015; Reyes-Cruz y Perales-Escudero, 2016). Incluso Filatov y Phil (2015) expresaron que la falta de experiencia profesionalizadora sobre conocimientos pedagógicos y de contenido repercute negativamente en el desarrollo profesional.

\section{Claves de futuro para los profesores y formadores}

$\mathrm{N}$ o importa si hablamos del profesor de la IVET o del formador de la CVET. Su desempeño o desarrollo profesional avanza en la misma dirección. Es decir, es un proceso de aprendizaje permanente donde confluye la formación inicial, el periodo de inserción en la profesión, la superación permanente y la autoformación con el fin de fortalecer las competencias específicas y transversales en el marco de una profesión en permanente construcción (Robalino, 2007, citado por Tejada, 2013).

En la sociedad actual, donde se quiere instaurar patrones para realzar la profesionalidad de los profesores y formadores, necesitamos promover la creación de estándares para la profesionalización y cualificación de los perfiles de la CVET y elevar el estatus y reconocimiento de los profesores y formadores en ambos sistemas (Haasler, 2012).

Es indudable que cada país sigue su estrategia para mejorar el desarrollo profesional de sus formadores y profesores, pues los enfoques y las medidas dirigidas a ellos siguen presentando alta diversificación y distinta regulación. Para consolidar el desarrollo profesional se necesita de una formación reconocida y reglada por los dos marcos europeos y como se estipula en los principios comunes europeos para las competencias y cualificaciones del profesorado:

Todos los profesionales de la educación/formación deben ser graduados en instituciones de educación superior; pues deben contar con amplios conocimientos de las materias que imparten, buena destreza pedagógica, capacidades y competencias necesarias para orientar y apoyar a los alumnos, y comprender la dimensión social y cultural de la educación.

(Comisión Europea, 2005 citado por Tejada 2013).

La profesionalización que se persigue es la combinación de dos procesos distintos y complementarios: el de la profesionalidad y el del profesionalismo (Lang, 1999). Mientras el primero es de carácter individual, referido a la 
persona como responsable de su propio desarrollo formativo, el segundo, el profesionalismo, refiere al colectivo que ejerce funciones específicas y considera las estrategias empleadas por ese grupo para transformar el estatus de su actividad a fin de, por ejemplo, obtener su reconocimiento social (Bourdoncle, 1991; Correa Molina, 2013). Esta dimensión colectiva juega un rol esencial en la construcción de la identidad del futuro profesional.

Avanzar en esta perspectiva contribuiría, en primer lugar, a mejorar la identidad profesional de ambos perfiles profesionales así como su desempeño; en segundo lugar, a concebir la docencia y la formación como una profesión que ocupa un espacio relevante y distinguido en el campo de actividades profesionales de la sociedad; en tercer lugar, prevendría y dificultaría el intrusismo profesional; $\mathrm{y}$, en cuarto lugar, establecería garantías para una tarea profesional de calidad (Bulgarelli, 2009).

\section{Notas}

1 Este estudio ha recibido el apoyo del proyecto de investigación LETEGR2, financiado por el General Secretariat for Research and Technology (GSRT) y la Hellenic Foundation for Research and Innovation (HFRI) (código de referencia: 1656).

2 Entendemos por Initial Vocational Education and Training (IVET) como la educación y formación inicial. Enseñanza o formación de tipo general o profesional impartidas dentro del sistema de la educación inicial, habitualmente antes de acceder a la vida activa. Notas: (a) algunas de las formaciones que se imparten una vez que la persona ingresa en la vida activa también pueden considerarse iniciales (p.e. en el caso de una reconversión); (b) la educación o formación inicial pueden tener lugar a travésde cualquier vía de enseñanza general o profesional (sea educación escolar de dedicación exclusiva o formación enalternancia) o a través de la formación de aprendices. Términos emparentados: educación obligatoria, formación incial. Fuente: adaptado de Cedefop, 2008.

3 Entendemos por Continuing Vocational Education and Training (CVET) como la formación que tiene lugar tras la educación o formación inicial -o tras acceder a la vida activa- y que pretende ayudar a las personas a:

- perfeccionar o actualizar sus conocimientos y/o destrezas;

- obtener nuevas destrezas para un nuevo puesto o una reconversión profesional;

- proseguir su desarrollo personal o profesional.

Términos emparentados: formación profesional para el empleo, aprendizaje a lo largo de la vida, educación de adultos, formación continua. Fuente: adaptado de Cedefop, 2008.

\section{Bibliografía}

American Council on Education. (1999). To Touch the Future: Transforming the Way Teachers Are Taught. An Action Agenda for College and University Presidents. Autoedición.

Andria, M. (2020). Learning to teach and teaching Greek as a Second/Foreign Language: A preliminary approach on teachers' and student-teachers' perceptions. En Mackay, J., Birello, M. y Xerri, D. (Eds.). (ELT Research in Action: Bringing together two communities of practice (pág. 43-46). IATEFL, Faversham, Kent.

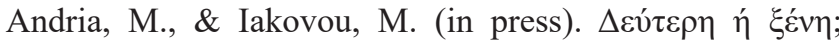

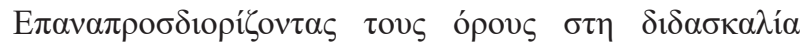

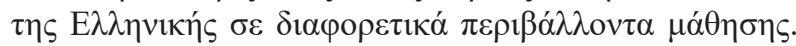
[Second or Foreign? Redefining the terms in the acquisition of Greek in different learning contexts]. Proceedings of the $14^{\text {th }}$ International Conference on Greek Linguistics (ICGL14), University of Patras, Patras, Greece.

ARMIF (2015). PATHWAY: Avaluació del projecte d'internacionalització en el Grau d'Educació Primàriaanglès UAB: procés i producte. (AGAUR: ARMIFF2015 REF00001)

Barber M. y Mourshed M. (2007). How the world's best performing school systems come out on top. McKinsey and Company.

Blignières-Légeraud, A. (2000). TTnet: trends in the development of training and the role of innovation as a transferable practice. (Report Dossier No 1). CEDEFOP.

Bourdoncle, R. (1991). La professionnalisation des enseignants: Analyses sociologiques anglaises et américaines. La fascination des professions. Revue française de pédagogie, (94).

https://doi.org/10.3406/rfp.1991.1368

Bulgarelli, A. (2009). Continuity, consolidation and change. Towards a European era of VET. (Report Reference series; 73). CEDEFOP.

CEDEFOP. (2008). Terminology of European education and training policy. A selection of 100 key terms. Office for Official Publications of the European Communities.

Cocca, M. y Cocca, A. (2016). Self-efficacy in pre-school and primary teachers: Does experience, educational background, and teaching level matter? $8^{\text {th }}$ International Conference on Education and New Learning Technologies, Barcelona.

https://doi.org/10.21125/edulearn.2016.0910 
Coiduras, J.L., Correa Molina, E., Boudjaoui, M., y Curto, A. (2017). Formación dual en el grado de educación: claves organizativas y pedagógicas. Qurriculum. Revista de teoría, investigación y práctica educativa, 30, 81102. https://www.ull.es/revistas/index.php/qurriculum/ article/view/38/22

Coiduras, J.L., París, G., Torrelles, C., y Carrera, X. (2014). La evaluación de competencias en una experiencia de formación dual de maestros: Diferencias y semejanzas entre tutores de escuela y de universidad. Estudios Pedagógicos, 40, (Especial 2014) 29-48.

https://doi.org/10.4067/s0718-07052014000200003

Comisión Europea (2005). Common European Principles for Teacher Competences and Qualifications. Official Journal of the European Union

Comisión Europea. (2007). Conclusions of the Council and of the Representatives of the Governments of the Member States, meeting within the Council of 15 November 2007, on improving the quality of teacher education. Official Journal of the European Union. (12 December 2007, C $300 / 07$, p. 6-9).

Comisión Europea. (2013). Eurypedia. Eurydice. https:// webgate.ec.europa.eu/fpfis/mwikis/eurydice/index. php?title=Home

Correa Molina, E. (2013). La Supervisión Clínica: Modalidad de Formación en Terreno. XII Simposio Internacional sobre el Prácticum y las Prácticas en Empresas den Formación Universitaria. Un Practicum para la formación integral de los estudiantes (págs. $37-$ 48). Andavira.

https://doi.org/10.35376/10324/4973

Cort, P., Harkonen, A. y Volmari, A. (2004). PROFF Professionalisation of VET teachers for the future. (Report Panorama series, 104).

Danielson, C. (2013). The Framework for Teaching. The Danielson Group.

EUCLID APPC (2008). European CLIL in Development: A primary phase consortium. European Commission (Education, Audiovisual y Culture Executive Agency).

Fernández Enguita, M., Mena, L. y Rivere, J. (2010). Fracaso abandono escolar en España (Colección Estudios Sociales, 29). La Caixa.

Filatov, K. y Phil, S. (2015). The relationship between university learning experiences and English teaching self-efficacy: Perspectives of five final-year pre-service English teachers. Australian Journal of Teacher Education, 40(6), 33-59.
García Molina, J.L. (2013). Profesionales de la enseñanza y la formación profesional. Revista Formación XXI, 21.

Goldhaber, D. y Brewerm, D. (2000). Does teacher certification matter? High school teacher certification status and student achievement. Educational Evaluation and Policy Analysis, 22, 129-145.

https://doi.org/10.3102/01623737022002129

Haasler, S. R. (2012). Tendencias de evolución en el escenario europeo. Revista Formación XXI, 21.

Hawes, G. y Corvalán, O. (2005). Construcción de un perfil profesional. Informe proyecto Mecesup. Tal0101. Universidad de Talca.

Hollingsworth, S.J. 1989. Prior beliefs and cognitive change in learning to teach. American Educational Research Journal, 26, 160-189.

https://doi.org/10.3102/00028312026002160

Iakovou, M. (En este volumen). Classroom observation in second language classrooms: Bridging the gap between theory and practice for pre-service and service teachers of Greek as an L2. CLIL Journal of Innovation and Research in Plurilingual and Pluricultural Education.

https://doi.org/110.5565/rev/clil.45

Lang, V. (1999). La professionnalisation des enseignants. Presses universitaires de France.

Marable, M., y Raimondi, S. (2007). Teachers' perceptions of what was most (and least) supportive during their first year of teaching. Mentoring and Tutoring: Partnership in Learning, 15, 25-37.

https://doi.org/10.1080/13611260601037355

Morris, D.B., y Usher, E.L. (2011). Developing teaching self-efficacy in research institutions: A study of awardwinning professors. Contemporary Educational Psychology, 36(3), 232-245.

https://doi.org/10.1016/j.cedpsych.2010.10.005

OECD (2019), PISA 2018 Results (Volume III): What School Life Means for Students'Lives, PISA, OECD Publishing. https://doi.org/10.1787/e26bc666-en

París, G. (2015). Los profesionales de la Formación Profesional para el Empleo: competencias y desarrollo profesional. (Tesis Doctoral, Universitat de Lleida). https://www.tdx.cat/bitstream/handle/10803/285529/ tgpm1de4.pdf? sequence $=2 \&$ is Allowed $=y$

https://doi.org/10.35376/10324/22300

https://doi.org/10.14221/ajte.2015v40n6.3 
París, G. Andria, M. y Escobar, C. (2017). Exploring Primary Education student- teachers' perceptions towards CLIL internships: a first approach. $19^{\text {th }}$ Annual International Conference on Education, 15-18 May 2017, Athens, Greece.

París, G. y Tejada, J. (2017). El desarrollo de los profesionales de la formación para el empleo: afectación de las trasformaciones estructurales, necesidades formativas, y factores de calidad. Revista Qurriculum: revista de teoría, investigación y práctica educativa, 30, 15-30.

https://doi.org/10.13042/bordon.2018.52351

París, G.; Tejada, J. y Coiduras, J. (2014). La profesionalización de los profesionales de la Formación para el Empleo en constante [in]definición en Europa. Revista de currículum y formación del profesorado, 18(2), 266-283. Universidad de Granada.

https://doi.org/10.13042/bordon.2018.52351

Reyes-Cruz, M. R y Gutiérrez Arceo, J. M. (2015). Sentido de autoeficacia en investigación de estudiantes de posgrado. Sinéctica, 45, 1-15.

Reyes-Cruz, M. R. y Perales-Escudero, M. D. (2016). Research self-efficacy and its sources in foreign language university faculty in Mexico: implications for educational policy. Higher Education Research and Development Journal, 35(4), 800-814.

https://doi.org/10.1080/07294360.2015.1137884

Robalino, M. (2007). Los docentes pueden hacer la diferencia; apuntes acerca del desarrollo profesional y el protagonismo docente. Séminaire International: La professionnalisation des enseignants de l'Education de base: Les recrutements sans formation initiale. Sèvres.

Sergiovanni, T. y Starratt, R. (2007). Supervision: A redefinition. McGraw Hill.

Tejada, J. (2009). Profesionalización docente en el escenario de la Europa del 2010. Una Mirada desde la formación. Revista de Educación, 349, 463-477.

Tejada, J. (2013). Profesionalización docente en la Universidad: implicaciones desde la formación. Revista de Universidad y Sociedad del Conocimiento, 10(1), 170-184.

Tejada, J.; Gimenez, V., Gan bustos, F.,Viladot. G., Fandos, M., Garrido, Pio, A. y Jimenez, J.M. (2007). Formación de formadores. Tomo 2. Escenario institucional. Ediciones Paraninfo.

Thorslund, J. y Jacobsen, J.C. (2006). ETUCE survey on trends in teacher education. ETUCE Conference. Brussels.
Tschannen-Moran, M. y Hoy, A. W. (2001). Teacher efficacy: Capturing an elusive construct. Teaching and Teacher Education, 17(7), 783-805.

https://doi.org/10.1016/s0742-051x(01)00036-1

Tschannen-Moran, M. y Hoy, A. W. (2007). The differential antecedents of self-efficacy beliefs of novice and experienced teachers. Teaching and Teacher Education, 23, 944-956.

https://doi.org/10.1016/j.tate.2006.05.003

Tutschner, R. y Kirpal, S. (2008). Eurotrainer: making lifelong learning possible: a study of the situation and qualifications of trainers in Europe. (Final report, Vol. 1). European Commission; Institute of Technology and Education.

Wilson, S., Floden, R., y Ferrini-Mundy, J. (2001). Teacher preparation research: Current knowledge, gaps, and recommendations: A research report prepared for the U.S. Department of Education. Centre for the Study of Teaching and Policy. 